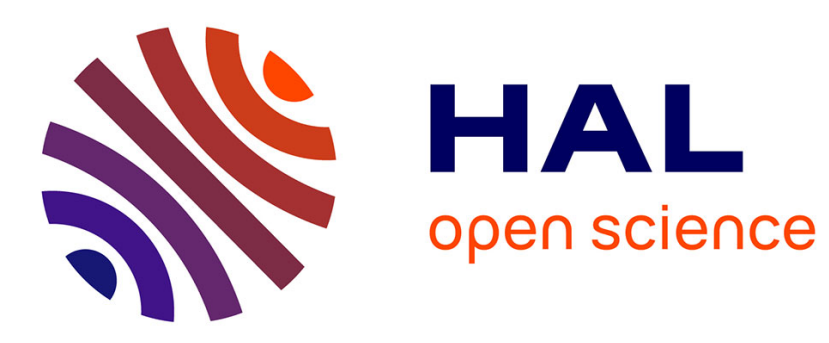

\title{
La piscine du soir, le dancing irlandais et les lieux vides...
}

Alain Faure, Elisabeth Sénégas

\section{To cite this version:}

Alain Faure, Elisabeth Sénégas. La piscine du soir, le dancing irlandais et les lieux vides.... L'Observatoire, la revue des politiques culturelles , 2018, Tiers-lieux : un modèle à suivre ?, 52, pp.5961. 10.3917/lobs.052.0059 . halshs-01828205

\section{HAL Id: halshs-01828205 https://shs.hal.science/halshs-01828205}

Submitted on 24 Jul 2018

HAL is a multi-disciplinary open access archive for the deposit and dissemination of scientific research documents, whether they are published or not. The documents may come from teaching and research institutions in France or abroad, or from public or private research centers.
L'archive ouverte pluridisciplinaire HAL, est destinée au dépôt et à la diffusion de documents scientifiques de niveau recherche, publiés ou non, émanant des établissements d'enseignement et de recherche français ou étrangers, des laboratoires publics ou privés. 


\title{
La piscine du soir, le dancing irlandais et les lieux vides...
}

\author{
Entretien entre Alain Faure et Élisabeth Sénégas
}

L'Observatoire nous a amicalement sollicités avec Élisabeth Sénégas pour raconter, sous la forme d'un entretien, les tiers-espaces qu'Élisabeth invente depuis vingt ans en Isère et dans la région grenobloise avec la création successive de l'association Entr'Actifs, de La Causerie et de La Chimère citoyenne. En premier réflexe, la commande nous a fait peur tant la thématique des tiers-lieux semble saturée de plaidoyers enflammés, d'analyses savantes et même de nouveaux dispositifs d'action publique. Mais Élisabeth s'est laissée convaincre (non sans mal) qu'il y avait peut-être un chemin de traverse à explorer en prenant la question à la racine, aux premières étincelles : celles de son plaisir et de sa détermination à «jouer collectif » à partir de lieux vides. Discussion à bâtons rompus...

Alain Faure - Quand je « couche » des élus sur le divan dans mes enquêtes de terrain (en tête-à-tête confidentiel), la première question porte toujours sur la politique avant la politique. Je cherche à retracer leur vision du monde en m'intéressant aux émois enfantins qui ont pu imprimer des blessures, des élans, des passions...

Élisabeth Sénégas - Ouh là, la psychanalyse, j'ai déjà donné et j'ai pas mal dégusté ! Mais si c'est pour la cause de la science... Je crois que ma rage de départ est directement liée à la façon dont mon père, qui était un entrepreneur, ne s'intéressait en apparence qu'aux exploits sportifs de mes frères ! J'étais extravertie, très agitée, boulimique de découvertes et toujours à la recherche des éclats que je pouvais provoquer dans le regard des autres. Et un jour, au bord d'une piscine, alors qu'on ne m'avait jamais appris à nager, un éducateur m'a encouragée à y aller. J'ai plongé (au propre et au figuré) et ça a transformé ma vie. J'étais euphorique, fière, pleine d'énergie. Je me suis même mise à la compétition. Je m'entrainais comme une forcenée. À 13 ans, j'ai quitté la maison pour un internat en sports-études. J'ai passé mon diplôme de maître-nageur. Et j'ai été jusqu'aux portes de l'équipe de France !

A. F. - Une thérapie de la confiance en soi par l'effort et la compétition?

E. S. - Non, c'est beaucoup plus compliqué que ça [rires]... J'ai fait plusieurs découvertes en même temps. Ma «vision du monde», pour reprendre ton jargon d'universitaire, s'est transformée avec la découverte que mon destin m'appartenait. En bossant dur, je pouvais avancer très vite et faire des vagues. Ma spécialité, c'était le papillon, une nage qui demande beaucoup de technicité et de fluidité. J'ai aussi constaté que je m'insérais naturellement dans un univers plein de ressources et qu'on m'appréciait pour ma facilité à nouer des relations d'amitié. Il y avait dans le club et dans les concours un brassage de gens très différents (des surdoués du sport, les bonnes familles annéciennes, des personnalités atypiques, des fêtards, des solitaires...). On se retrouvait tous au bord de la piscine sur une sorte d'égalité imparable : juste avec un maillot de bain. J'existais et les gens m'appréciaient pour ce que j'étais. Pour terminer là-dessus (on y a passé plusieurs années avec mon psychanalyste), j'ai perdu mon père à 23 ans, il n'est jamais venu à une 
seule compétition alors qu'il allait supporter mes frères tous les week-ends au rugby. Ça m'a donné une rage de vivre qui me surprend encore aujourd'hui...

A. F. - La blessure des injustices sur le statut garçon/fille?

E. S. - Beaucoup plus positif que ça : l'euphorie de constater qu'autour de la piscine, un lieu vide par excellence (juste de l'eau et une odeur entêtante de javel), on pouvait refaire le monde et le voir différemment. Un peu plus tard, quand j'étais conseillère d'éducation, j'ai convaincu le proviseur pour qu'il me donne les clefs de la piscine de quartier et, une fois par semaine, le soir, j'embarquais une quinzaine de gamins. J'ai senti très concrètement à quel point le collectif dans un lieu neutre, ça pouvait être puissant, joyeux, émancipateur, à quel point ça permettait de rire et de grandir ensemble. En passant, j'ai compris que je n'avais pas l'esprit de compétition au sens guerrier du terme, et c'est sans doute ce qui m'a fait rater la marche des compétitions internationales. On peut sortir de la piscine maintenant?

A. F. - Oui, mais il faut que tu m'éclaires sur les raisons pour lesquelles tu «plonges » ensuite dans la création de tiers-espaces. Je pense bien sûr à la Maison commune que tu as imaginée à Voiron, mais aussi à La Causerie puis à La Chimère citoyenne depuis trois ans à Grenoble.

E. S. - Tu te souviens qu'on a projeté le film Jimmy's Hall de Ken Loach au Méliès pour fêter les dix-huit mois d'expérimentation de La Chimère. Ça répond en images à ta question : le bonheur éprouvé autour de la piscine, c'est le même que celui de ce village irlandais qui décide, contre vents et marées, de retaper une grande ferme pour en faire un dancing foutraque et débridé où tout s'apprend et se réapprend, de la couture au piano, de la bibliothèque à l'atelier menuiserie, du bal aux délibérations politiques. On construit ensemble un espace où il devient possible de traiter les problèmes de souffrance sociale qui n'entrent pas dans les cases connues (souvent exiguës) des dispositifs d'action publique ou des associations caritatives. Ma conviction, c'est que les individus doivent d'abord reconquérir leur dignité autour de projets qui les touchent intimement. Il faut pour cela un lieu neutre, un point de rencontre particulier où il redevient possible de débroussailler ensemble des objectifs de confiance et de fierté. La piscine de mon enfance, c'était une atmosphère grisante de renaissance, tout comme le dancing de Jimmy's Hall. La Chimère citoyenne est sur la même orbite!

A. F. - Ce qui m'intrigue dans cette atmosphère de renaissance, c'est la texture mystérieuse de l'esprit des « lieux chimériques ». Pourquoi insistes-tu tant, par exemple, sur l'importance d'un « lieu vide »?

E. S. - J'ai mis longtemps à comprendre que les plus belles rencontres passaient par un espace ouvert et surtout qui ne soit ni « institué » ni « orienté». Il faut accepter l'inattendu, il faut accepter de prendre le risque de la relation à l'autre sans savoir où ça nous mène à priori. D'ailleurs, ça mène toujours quelque part, mais rarement là où l'on pensait. Dans la mode actuelle du coworking, on se fait vite piéger par des formats qui ne sont pas ouverts à tous et qui ne sont pas neutres. Je préfère d'ailleurs le terme de tiersespace à celui de tiers-lieu. La Chimère citoyenne, ce n'est pas un café anonyme, c'est une pièce chaleureuse, comme chez soi mais sans l'entre-soi, où l'on va chercher certains publics qui ne viendraient 
pas spontanément. On bricole, on invente, on recrée des relations, tout est possible, l'imaginaire de chacun s'articule à quelque chose de plus collectif...

A. F. - L’esprit de Mai 68 et de ses lieux alternatifs, sans règles, autogérés...

E. S. - Non, non! Je ne nous situe pas du tout sur ce registre idéologique et nostalgique. Pour faire tourner le local, il faut de la poigne et parfois des prises de décision sans discussion collective. L'objectif visé, c'est de favoriser un lieu de brassage de publics et de gens concernés par le réel. La première chose qui nous mobilise à La Chimère, c'est la relation humaine, pas un catalogue d'activités ou des promesses de grand soir. Par exemple, on n'est pas du tout dans l'amélioration technique des relations services publics/usagers. On ne brandit pas non plus « convivialité », " bienveillance », " participation » et tout le blabla pour rendre les services publics et les entreprises plus «efficaces», plus « solidaires», plus « durables ». Il faut du temps, beaucoup de temps et une grande qualité d'accueil. On construit un lieu qui donne envie de s'y installer... La tendance actuelle est plutôt à la multiplication des espaces de circulation impersonnels où prévaut l'anonymat, avec la formation de petites tribus. Ça peut paraître grandiloquent, mais La Chimère est un espace de résistance pour redécouvrir les fondements humains comme le don et la confiance, la capacité à penser par soi-même.

\section{A. F. - C'est un programme politique!}

E. S. - Ça, c'est au chercheur en sciences politiques de le dire... Ce qui est sûr, c'est qu'on est à contrecourant par rapport aux dispositifs classiques de l'intervention publique, qu'ils soient descendants ou ascendants. Pas par esprit de contradiction, mais parce que ces dispositifs nous renvoient systématiquement à des filières, à des procédures, à des grilles d'évaluation, à des thématiques. Avec dix ans d'expériences, on a fait le tour des différentes filières de subvention qui sont proposées. Il y a presque toujours un malentendu avec les financeurs qui donnent leur bénédiction sur des critères très restrictifs et souvent « ciblés » sur leurs propres publics. Quand on leur demande de nous accompagner pour le fonctionnement de ce lieu vide, les élus et les techniciens nous répondent " politique culturelle », " aide à la réinsertion », « transition écologique», «formation continue », « développement social», « accompagnement individualisé », etc. Notre "public » à nous est polymorphe : jeunes, vieux, riches, pauvres, étudiants, de droite, de gauche, malades, à la retraite, associations, groupes spontanés, etc. On est aussi à contre-courant de la posture du « professionnel accompagnant» dans la mesure où les attitudes relationnelles s'acquièrent au fil des rencontres par l'expérience et par la confrontation.

A. F. - N'est-ce pas un peu facile de déplorer la professionnalisation et la spécialisation des interventions proposées par les institutions?

E. S. - On ne le déplore pas, on dit simplement que ça ne colle pas avec notre façon de traiter les sollicitations et les demandes, qu'elles soient individuelles ou collectives. Je passe une dizaine d'heures par semaine en rendez-vous dont on ne peut pas imaginer la diversité : la recherche d'un job, des projets de création, une fédération nationale en quête d'idées, l'appel au secours d'un jeune, la parution d'un essai, un migrant mis en lien avec nos réseaux, une association sans local, un logement solidaire, un événement 
culturel... Si tu vas sur le site internet de La Chimère ${ }^{1}$, tu es tout de suite déstabilisé par la diversité des expériences en présence. Nous animons des ateliers collectifs sur le bien vivre, la bio-éthique, le logement précaire, le burn out, l'autisme, le micro-entrepreneuriat, la poésie, les personnes isolées, les arts de l'attention...

A. F. - Que faut-il attendre des « pouvoirs publics » dans ces conditions ?

E. S. - Qu'ils nous aident à trouver un local en centre-ville ! Et à payer une partie du loyer jusqu'à ce que le Chimère Café équilibre tout seul son budget. Sans demander pour autant d'orienter ce tiers-espace dans une case en conditionnant leur soutien à des « résultats » codifiés par l'administration. Le Département, qui nous a soutenus au départ, n'a souhaité continuer le partenariat que si nous limitions nos activités à l'insertion sociale. La Métropole ne propose que des aides ciblées sur des publics et des thématiques spécifiques (jeunes, politique de la ville, économie sociale et solidaire, équipements culturels...). La Ville conditionne son soutien à des «votations citoyennes», obnubilée par les micro-projets de quartier. Paradoxalement, ce sont les partenaires privés (la Fondation Veolia, AG2R-La Mondiale, des donateurs) qui comprennent le mieux notre démarche et qui nous proposent des financements d'investissement sans contrepartie chiffrée ou ciblée. Avec nos parrains historiques (Edgar Morin et Jean-Paul Delevoye), avec nos compagnons de l'université, avec ceux du monde de l'entreprise, avec des artistes et des écrivains, avec des lycéens de Champollion, avec notre réseau de 35 associations et groupes et, bien sûr, avec nos 400 chiméristes qui donnent et prennent régulièrement des nouvelles, nous nous démenons pour que le projet de Chimère Café préfigure un dancing vivant et intrépide au cœur de la métropole grenobloise. On est en maillot de bain et on invente une belle piscine en centre-ville pour que les cabossés de la vie, que nous sommes tous, apprenions à nager ensemble...

Entretien entre Alain Faure CNRS - Sciences Po Grenoble

et

Élisabeth Sénégas

La Chimère citoyenne

${ }^{1}$ https://lachimerecitoyenne.org/ 